\title{
Adult Neuron Addition to the Zebra Finch Song Motor Pathway Correlates with the Rate and Extent of Recovery from Botox-Induced Paralysis of the Vocal Muscles
}

\author{
Carolyn Pytte, ${ }^{1}$ Yi-Lo Yu, ${ }^{2}$ Sara Wildstein, ${ }^{1,3}$ Shanu George, ${ }^{1}$ and John R. Kirn ${ }^{4}$ \\ ${ }^{1}$ Queens College, City University of New York, Flushing, New York 11367, ${ }^{2}$ Hartford Hospital, Hartford, Connecticut 06102, ${ }^{3}$ Macaulay Honors College, City \\ University of New York, New York, New York 10023, and ${ }^{4}$ Wesleyan University, Middletown, Connecticut 06459
}

In adult songbirds, neurons are continually incorporated into the telencephalic nucleus HVC (used as a proper name), a premotor region necessary for the production of learned vocalizations. Previous studies have demonstrated that neuron addition to HVC is highest when song is most variable: in juveniles during song learning, in seasonally singing adults during peaks in plasticity that precede the production of new song components, or during seasonal reestablishment of a previously learned song. These findings suggest that neuron addition provides motor flexibility for the transition from a variable song to a target song. Here we test the association between the quality of song structure and HVC neuron addition by experimentally manipulating syringeal muscle control with Botox, which produces a transient partial paralysis. We show that the quality of song structure covaries with new neuron addition to HVC. Both the magnitude of song distortion and the rate of song recovery after syringeal Botox injections were correlated with the number of new neurons incorporated into HVC. We suggest that the quality of song structure is either a cause or consequence of the number of new neurons added to HVC. Birds with naturally high rates of neuron addition may have had the greatest success in recovering song. Alternatively, or in addition, new neuron survival in the song motor pathway may be regulated by the quality of song-generated feedback as song regains its original stereotyped structure. Present results are the first to show a relationship between peripheral muscle control and adult neuron addition to cortical premotor circuits.

\section{Introduction}

The neural substrate for birdsong learning and production includes a series of interconnected telencephalic nuclei termed the "song system" (Fig. 1). Within the song system, HVC (used as a proper name) and Area X continue to integrate new neurons throughout life, providing a model for the study of adult neurogenesis in relation to a well-defined behavior. In HVC, new neurons project to the premotor robust nucleus of the arcopallium (RA; Alvarez-Buylla et al., 1990; Scotto-Lomassese et al., 2007), becoming part of the motor pathway for song production.

HVC neurons are generated in the ventricular zones lining the lateral ventricles, begin to reach HVC within 1 week, and by 15-40 d after formation, $50 \%$ have died (Kirn et al., 1999). Neurons that survive this period of early culling persist for at least 4-8 months (Nottebohm et al., 1994). In canaries, the number of

Received June 13, 2011; revised Sept. 20, 2011; accepted Sept. 29, 2011

Author contributions: C.P., Y.-L.Y., and J.R.K. designed research; C.P., Y.-L.Y., S.W., and S.G. performed research; C.P., Y.-L.Y., S.W., and S.G. analyzed data; C.P. and J.R.K. wrote the paper.

This work was supported by PSC-CUNY Award PSCOOC-39-1018 and National Institutes of Health Grants R03 41684 (C.L.P.) and DC004724 (J.R.K.). We gratefully acknowledge Dr. Roger Aoki and Perla Baker at Allergan for supplying Botox. We thank Natalia Gonzalez for her contribution to behavioral data, Barry Chernoff for statistical advice, and Joshua Brumberg for comments on previous versions of this manuscript.

The authors declare no competing financial interests.

Correspondence should be addressed to Carolyn Pytte, Psychology Department, Queens College, City University of New York, 65-30 Kissena Boulevard, Flushing, NY 11367. E-mail: carolyn.pytte@qc.cuny.edu.

DOI:10.1523/JNEUROSCI.2971-11.2011

Copyright $\odot 2011$ the authors $\quad 0270-6474 / 11 / 3116958-11 \$ 15.00 / 0$ new HVC neurons is positively correlated with the amount of singing (Li et al., 2000; Alvarez-Borda and Nottebohm, 2002). This effect may be mediated by brain-derived neurotrophic factor (BDNF), which is upregulated by singing (Rasika et al., 1999; Li et al., 2000) and which prolongs the lifespan of new HVC neurons when present during a critical period of neuron age (Alvarez-Borda et al., 2004). However, on a larger timescale, seasonal peaks in new neuron addition correspond to low singing rates during periods when song is variable (Alvarez-Buylla et al., 1990; Kirn et al., 1994), supporting the proposal that singing rate may be only one song-related attribute modulating HVC neuron addition (Alvarez-Borda and Nottebohm, 2004).

A consistent pattern across species and contexts is that neuron addition to HVC is highest when birds' songs are variable and indistinct and diminishes as song becomes stereotyped. This association between increasing song stereotypy and decreasing neuron incorporation is seen in juvenile song learning, seasonal song changes, and as adult birds age (Kirn et al., 1994; Tramontin and Brenowitz, 1999; Wilbrecht et al., 2002, Pytte et al., 2007). Here we experimentally tested this association by reversibly altering song structure, thereby shifting the song "off target," and examining whether there is a relation between song recovery and new HVC neuron recruitment. To do this, we injected botulinum toxin (Botox) into the vocal muscles of the syrinx, inducing a transient partial paralysis, which distorted song structure but did not prevent singing. We also assessed potential relationships between amount of singing and new neuron incorporation in the 
zebra finch, a species that, unlike the canary, sings at comparable rates throughout the year.

Our results show that birds with the least song distortion had the highest rate of song recovery and also the highest levels of neuron addition. Amount of singing showed no association with levels of neuron addition. We propose a functional relation between neuron addition and the achievement of a target song. New neurons may contribute to song recovery, and/or increasingly accurate song may promote new neuron survival.

\section{Materials and Methods}

\section{Animals}

This study was consistent with National Institutes of Health and institutional animal use guidelines. All birds were hatched in the Wesleyan breeding colony and kept with their parents until 70-90 d after hatching. Thereafter, birds were housed in all-male groups of $7-10$, within auditory and visual contact of birds of both sexes, until 1 month before cell birth dating. Birds were then housed individually in cages that provided visual and acoustic contact with other birds throughout the experiment and maintained on a $13 / 11 \mathrm{~h}$ light/dark cycle. Subjects $(n=24)$ were adult male zebra finches $175-239 \mathrm{~d}$ at time of cell birth dating (mean, $208 \pm$ 3 d, i.e., 7 months).

\section{Cell birth dating}

Mitotically active cells were labeled with intramuscular injections of bromodeoxyuridine (BrdU; $75 \mu \mathrm{l}$ of a $10 \mathrm{mg} / \mathrm{ml}$ solution in $0.1 \mathrm{M}$ Trisbuffered saline, $\mathrm{pH}$ 7.4; Roche Diagnostics) three times per day for 4 consecutive days.

\section{Botox injections}

Three to $4 \mathrm{~d}$ after the last BrdU injection, experimental birds $(n=11)$ received intrasyringeal injections of botulinum toxin (Botox; Allergan) as described by Pytte and Suthers (2000), using ketamine (0.03$0.05 \mathrm{mg}$ of ketamine per gram body weight of bird) and xylazine $(0.06$ $\mathrm{mg} / \mathrm{g}$ xylazine) anesthesia. Briefly, $100 \mathrm{MU}$ of Botox was reconstituted in $330 \mu \mathrm{l}$ of $0.1 \mathrm{M}$ PBS. One microliter of reconstituted Botox solution, containing 0.33 MU of Botox, was gently pressure-injected bilaterally into the ventral and ventrolateral muscles of the syrinx. Control birds $(n=13)$ received anesthesia, a skin incision, and sutures. Syringeal manipulation was not conducted in control birds because opening the interclavicular air sac surrounding the syrinx may result in aberrations in vocalizations as a result of changes in the properties of air sac membranes. Cell birth dating before Botox injections ensured that any effects of the Botox treatment on labeled neuron number would be attributable to alterations in postmitotic events and not changes in neuron proliferation.

\section{Histology}

Four weeks after the final BrdU injection, birds were perfused transcardially with PBS, pH 7.4, followed by $4 \%$ paraformaldehyde, $\mathrm{pH} 7.4$. The brains were postfixed for $1 \mathrm{~h}$ in the same fixative, rinsed in PBS for $2-3 \mathrm{~h}$, dehydrated in increasing concentrations of ethanol, and embedded in polyethylene glycol (Polysciences). Six micrometer sagittal brain sections were cut on a rotary microtome, and every eighth section was mounted onto slides (Superfrost ++ ; Electron Microscopy Sciences) and stored at $-20^{\circ} \mathrm{C}$.

\section{Immunohistochemistry}

Sections were brought to room temperature and exposed to citrate buffer at $95^{\circ} \mathrm{C}$ for $10 \mathrm{~min}$, followed by a $5 \mathrm{~min}$ wash in $0.1 \mathrm{M}$ phosphate buffer (PB), $3 \mathrm{~min}$ in $2.5 \%$ pepsin in $0.1 \mathrm{~N} \mathrm{HCl}$ at $37^{\circ} \mathrm{C}$, and three 3 min washes in PB. Sections were then blocked with $10 \%$ normal donkey serum (Jackson ImmunoResearch) and $0.3 \%$ Triton X-100 in PB for $1 \mathrm{~h}$ at room temperature, followed by overnight exposure to sheep anti-BrdU (12.5 $\mu \mathrm{g} / \mathrm{ml}$ at $4^{\circ} \mathrm{C}$; Capralogics). After three $10 \mathrm{~min} \mathrm{~PB}$ washes, sections were processed with an avidin-biotin blocking kit (Vector Laboratories), followed by a $2 \mathrm{~h}$ incubation in biotin-conjugated donkey anti-sheep IgG (1:200; Millipore Bioscience Research Reagents). After three $10 \mathrm{~min}$ PB washes, streptavidin conjugated to Alexa Fluor $488(1.25 \mu \mathrm{g} / \mathrm{ml}$; Invitro-

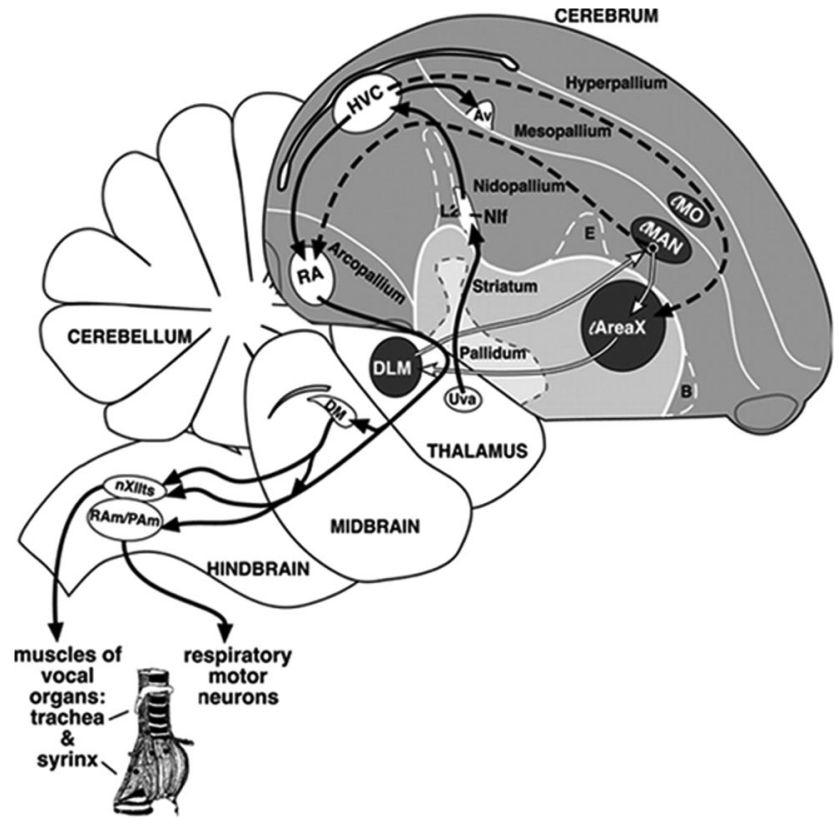

Figure 1. Diagram of the song system. Solid black lines show the pathway necessary for the production of song. Dashed and white lines together show the anterior forebrain pathway necessary for song learning and adult song maintenance. Within the song system, only HVC and Area $X$ receive new neurons in adulthood. Av, Nucleus avalanche; $B$, nucleus basorostralis; DM, dorsal medial nucleus; DLM, dorsal lateral nucleus of the medial thalamus; $E$, entopallium; HVC (used as a proper name); LMAN, lateral magnocellular nucleus of the anterior nidopallium; LMO, lateral oval nucleus of the mesopallium; NIf, interfacial nucleus of the nidopallium; RA, robust nucleus of the arcopallium; RAm, nucleus retroambiguus; Uva, nucleus uvaeformis; Area X, Area $X$ of the medial striatum; nXIIts, tracheosyringeal part of the hypoglossal nucleus. (Reproduced with permission Wilbrecht and Kirn, 2004.)

gen) was applied for $1 \mathrm{~h}$ in the dark for visualization of BrdU. All subsequent incubations took place in the dark when feasible. Tissue was then washed three times for $10 \mathrm{~min}$ in $\mathrm{PB}$, incubated for $1 \mathrm{~h}$ in blocking solution, and overnight in mouse anti-Hu primary antibody $(10 \mu \mathrm{g} / \mathrm{ml}$ in blocking solution) ( $\mathrm{Hu}$ MAB16A11; Invitrogen) at $4^{\circ} \mathrm{C}$. After three 10 min $\mathrm{PB}$ washes at room temperature, tissue was then exposed to donkey anti-mouse IgG conjugated to Cy3 $(6.25 \mu \mathrm{g} / \mathrm{ml}$; Jackson ImmunoResearch) for $1 \mathrm{~h}$ to visualize labeling of the cytoplasmic neuron-specific $\mathrm{Hu}$ protein. Sections were then washed, dehydrated in ethanols, immersed briefly in xylenes, and coverslipped with Krystalon (Harleco; EM Science).

\section{Microscope analysis}

Data were collected without knowledge of bird identity. Area measurements and cell counts were performed using a computer-yoked fluorescence microscope (Olympus BX50 and Olympus BX51; Neurolucida from MicroBrightField). Boundaries for HVC were established with dark-field optics based on neuropil density and contrast. HVC areas in 10 sections distributed evenly throughout HVC were measured, and BrdUpositive cells were counted. It was noted whether these cells were also labeled for $\mathrm{Hu}$. Anti-BrdU-labeled $(\mathrm{BrdU}+)$ cells were identified with a fluorescein isothiocyanate (FITC) filter and anti-Hu-labeled cells with a rhodamine filter. Double-labeled cells were identified by alternating between these two filters and also using a dual FITC-rhodamine filter. The total number of $\mathrm{Hu}$-expressing $\left(\mathrm{Hu}^{+}\right)$cells that contained a nucleus and at least one nucleolus was counted in five evenly spaced sections throughout HVC. In each of these sections, the entirety of HVC was examined, and all Hu-expressing cells meeting these criteria were counted. Cell nuclear size changes of the magnitude encountered here are not likely to bias estimates of neuron number derived from $6 \mu \mathrm{m}$ sections (Clark et al., 1990); therefore, no corrections were made for cell splitting because we were primarily interested in relative differences in neuronal number and not absolute values. 
Cross-sectional areas throughout HVC were multiplied by section thickness and sampling interval to estimate HVC volumes (as in the study by Kirn et al., 1991). Cell densities per square millimeter were calculated by dividing the number of cells in each label category by the area sampled. Estimates of total new neuron number in HVC were calculated by multiplying the density of $\mathrm{BrdU}^{+} / \mathrm{Hu}^{+}$cells per sampled volume by the total estimated HVC volume. The percentage of new neurons of the total number of neurons was calculated as the number of $\mathrm{BrdU}^{+} / \mathrm{Hu}^{+}$cells divided by the number of $\mathrm{Hu}^{+}$cells multiplied by 100 . As with $\mathrm{Hu}^{+}$cell counts, no corrections were made for cell splitting because we were interested in relative rather than absolute differences in neuronal number (Saper, 1996; Guillery and Herrup, 1997). Multiple measurements of labeled neurons were used because they are not necessarily redundant and provide a more thorough assessment of treatment effects than any single measure (see discussion by Kirn and DeVoogd, 1989).

\section{Song recording}

All birds were housed individually and recorded continuously for at least $7 \mathrm{~d}$ before Botox or sham treatment. Experimental birds were recorded for $24 \mathrm{~h}$ on post-Botox days 7-8, 14-15, and 27-28 using Avisoft SASLab Pro Recorder (Avisoft Bioacoustics). Control birds were recorded for the same duration and at the same intervals $\pm 3 \mathrm{~d}$. Birds were in visual and acoustic contact with other males in the same room and therefore songs recorded were either undirected or directed toward males. No females were present in the room.

\section{Analysis of song structure}

Selection of motifs for analysis. Zebra finch songs are composed of a variable number of repetitions of an introductory note or syllable, followed by a sequence of usually $4-10$ syllables produced in a consistent order, termed a motif. A syllable is defined as an acoustically unique sound element, seen as a continuous sound on a spectrograph. "Song" is a more general term, which may refer to motifs with or without introductory notes. We selected 30 motifs produced by each experimental bird at each of the recording sessions: 2 pretreatment days, post-Botox day 7, postBotox day 14, and post-Botox day 27. Thirty motifs produced by each control bird were selected on 2 pre-sham days and on one day 20-27 days later. Motifs were selected for analysis based on recording quality, song completeness, and the requirement that songs were produced later than $1 \mathrm{~h}$ after lights turned on to avoid a potential early morning period of increased song variability (Deregnaucourt et al., 2005). Because HVC-RA projection neurons appear to code motor commands on a fine temporal scale (Hahnloser et al., 2002), we were interested specifically in potential correlations between acoustic fine structure and new HVC neurons. Therefore, motifs were selected to be identical in syllable sequence to compare the structure of like-syllables across motifs and to quantify song distortion based solely on syllable acoustic structure and not syllable sequence (as in the study by Pytte et al., 2007). In our quantitative analysis (described below), we loaded the syllables into Sound Analysis Pro (version 1.02; Tchernichovski et al., 2000) embedded in situ in the motifs. Because the motifs were identical in sequence, the program compared the similarity of the same syllables before and after treatment and did not compare similarity in structure across different syllable types. This was the case except for two birds for which post-Botox songs were too different from pre-Botox songs to be aligned (Fig. $2 A, B$ ). For these birds, we still used their post-Botox motifs in our comparison with pre-Botox motifs. The similarity threshold setting in Sound Analysis Pro was set low enough so that the program selected the best case pre-syllable and postsyllable matches and output an accuracy score based on the most similar syllables. Omission of these two birds from our analyses did not change the results.

Qualitative song structure analysis. To qualitatively assess song recovery after Botox injections, we visually examined the songs of experimental birds using spectrograms generated by Raven Pro 1.3 (Cornell Lab of Ornithology) and Avisoft SASLab Pro. For each bird, we printed five representative spectrograms of pre-Botox songs and post-Botox songs at day 27 (10 total for each bird). We aligned pre- and post-Botox songs and scored post-Botox syllable morphology on a scale of 0 [no difference (fully recovered)] to 4 (post-Botox syllable that is unrecognizable from the pre-Botox syllable) (modified from Williams and McKibben, 1992; Pytte and Suthers, 2000). The syllable scores were averaged for each bird, and then birds were ranked according to overall recovery score. Songs were analyzed independently by two experienced observers (S.G. and C.P.). We assessed inter-observer reliability using Kendall's tau, then averaged the rank scores between the two observers, and compared the mean scores of completeness of recovery with the measures of new HVC neurons using Kendall's tau correlation.

Quantitative song structure analysis. Quantification of acoustic changes in song structure was conducted using Sound Analysis Pro (version 1.02; Tchernichovski et al., 2000). Sound Analysis Pro version 1.02 provides a single unit-less score of the accuracy between two sounds based on a comparison of four acoustic features (Wiener entropy, spectral continuity, pitch and frequency modulation) across shifting $50 \mathrm{~ms}$ time frames of sound centered at successive $7 \mathrm{~ms}$ intervals. The output of these comparisons is a mean accuracy score that represents the accuracy of the match between these acoustic features across sequential time frames throughout the sounds being compared. We refer to this score as the "accuracy score."

We used Sound Analysis Pro to compare two sets of 30 motifs at a time (described further below). For each comparison, we used the matrix function of Sound Analysis Pro, which compares each motif to all others in the set. We excluded duplicate comparisons so that members of each pair of songs were compared with each other only once. The output of this matrix analysis provided a mean accuracy score among the 30 motifs. Symmetrical analyses were used rather than asymmetrical analyses (two options in Sound Analysis Pro). Symmetrical analysis provides a better representation of the acoustic changes after Botox when syllables are added, deleted, or significantly changed from the pre-Botox syllables and thus best captures changes in acoustic structure under all of our conditions.

Individual song stereotypy. Before measuring the effects of Botox on song acoustic structure, we first quantified the degree of naturally occurring song stereotypy within experimental individuals before the Botox injections. To do this, we compared 30 pre-Botox motifs taken from a single day with 30 songs taken from a second pre-Botox day that was separated from the first by $3-5 \mathrm{~d}$.

For each bird, the mean of these accuracy scores is the pre-to-pre accuracy score, a measure of individual song stereotypy. We normalized the Botox-induced changes in acoustic structure to these pre-Botox values of motif variation.

The same procedure was performed using songs of control birds to determine whether song stereotypy within controls changed over the course of the experiment. We computed song stereotypy between a set of 30 motifs taken from a single day with 30 motifs recorded 3-5 d later (control pre-to-pre) and also with a set of 30 motifs recorded $20-27 \mathrm{~d}$ later (control pre-to-post). There were no differences in these measures of stereotypy (repeated-measures ANOVA, $p>0.05$ ).

Song distortion after Botox injections. We compared one of the sets of 30 pre-Botox motifs that were used to quantify pre-Botox song stereotypy with three sets of 30 post-Botox motifs: recorded on days 7, 14, and 27 (resulting in three pre-to-post accuracy scores per individual). This provided a measure of song distortion at each of these sampling times.

For each bird, the pre-to-post accuracy scores were subtracted from the pre-to-pre accuracy score to obtain measures of song distortion relative to the bird's natural baseline pre-Botox song variability. This song distortion score was then subtracted from 100 to present the score as a normalized accuracy score. A low pre-post accuracy score indicated high distortion after Botox injection.

For example, the pre-to-pre accuracy score may be 90, as opposed to the theoretical maximum accuracy of 100 if all songs were identical. A comparison of 30 pre-Botox songs and 30 post-Botox songs may produce an accuracy score of 20 (the pre-to-post accuracy score). We would then subtract the pre-to-post accuracy score of 20 from the pre-to-pre accuracy score of 90 , resulting in a distortion value of 70 . The score of 70 is then subtracted from 100, resulting in 30 as the normalized pre-post accuracy score, as opposed to the non-normalized accuracy score of 20. All data are presented as normalized accuracy scores. 
A Bird 44: Song nearly stabilized Song changed, d27 AS $=31.6$

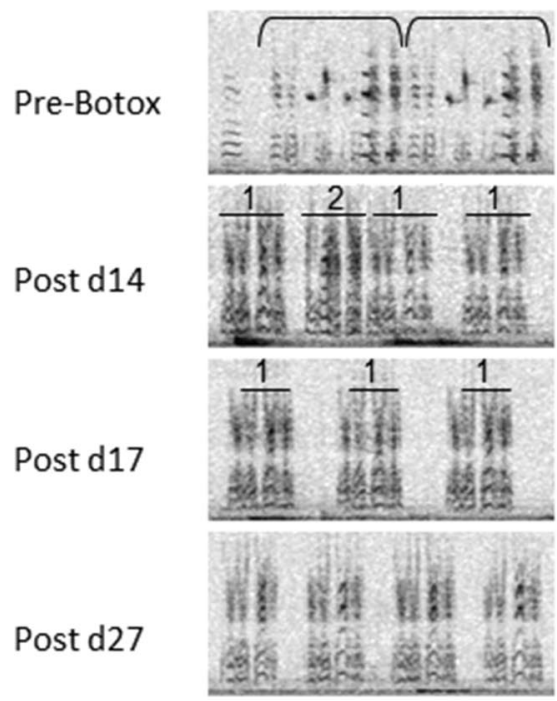

C Bird 65: Song not stabilized Original song recovering, $\mathrm{d} 27 \mathrm{AS}=70.4$

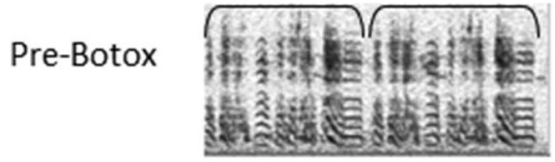

Post d14

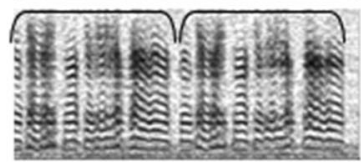

Post d17

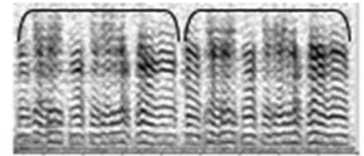

Post d 27

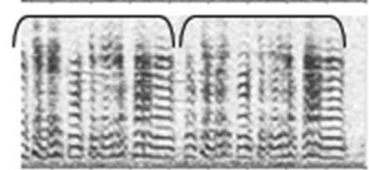

\section{B Bird 181: Song stabilized \\ Song changed, d27 AS=5.7}
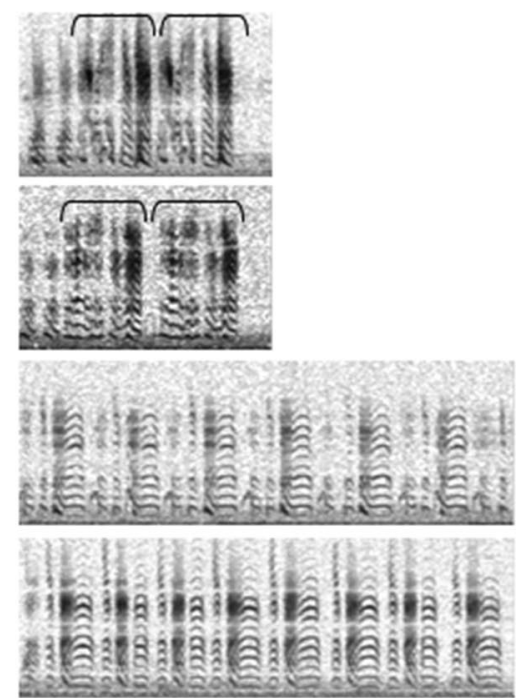

D Bird 47: Song stabilized

Original song largely recovered, $\mathrm{d} 27 \mathrm{AS}=87.6$
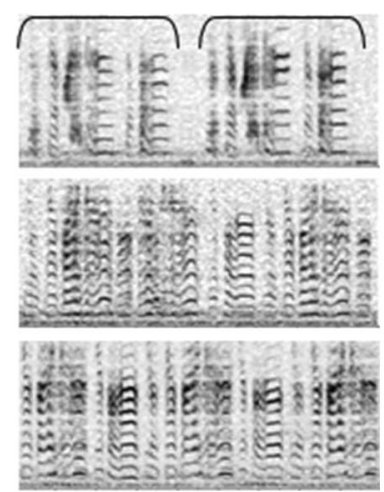

$8 \mathrm{kHz}$

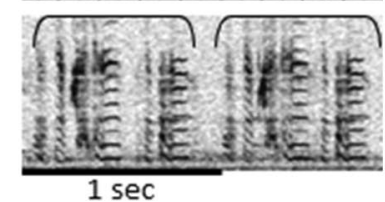

Figure 2. Spectrographs of the songs of four birds before Botox injections and on days 14,17 , and 27 after Botox injections illustrate varying degrees of song recovery. Brackets indicate song motifs. A, At day 14 after Botox, bird 44 had no easily recognizable matches to pre-Botox note structure. Between post-Botox days 14 and 17 , note that cluster 2 was dropped from the repertoire. There were no other major changes in the song structure between days 17 and 27 . By day 27 , the effects of Botox were primarily worn off, based on subjective assessment of stereotypy of acoustic fine structure seen in spectrographs. However, the motifs were not yet completely stabilized, which would be evident in a larger sample of motifs. To a lesser degree, instability in the motifs can be seen by comparing the small structural differences in the four motifs shown in the post-Botox day 27 panel. The final stereotyped song was considerably changed from the pre-Botox song. Accuracy scores (AS) to pre-Botox song: day 14, 28.2; day 17, 24.7; day 27, 31.6. B, The song temporal pattern and acoustic structure of bird 181 changed between post-Botox days 14 and 17 so that the motifs at day 17 could no longer be aligned with the pre-Botox song. Accuracy scores to pre-Botox song: day 14, 13.4; day 17, 12.7; day 27, 5.7. This is an example of a negative accuracy score (or "negative recovery") as song became less similar to pre-Botox song between postoperative days 14 and 27. C, Bird 65 retained its pre-Botox temporal pattern throughout the survival period; however, the effects of Botox were still apparent at day 27. These effects included variability in note structure (across song renditions within $1 \mathrm{~d}$ and from one day to the next), acoustic structure composed primarily of harmonic stacks, and lack of stereotyped frequency modulation. The lack of stereotypy is not apparent in the sample of two motifs shown in the post-Botox day 27 panel but would be seen in a larger song sample. Accuracy scores: day 14,62.2; day 17,59.1; day 27,70.4. D, Bird 47 primarily regained its pre-Botox song acoustic structure and temporal pattern by post-Botox day 27 . Fine acoustic structure and temporal pattern were stabilized. Accuracy scores: day 14,30.2; day 17, 42.7; day 27, 87.6. Spectrographs of recordings from day 17 after Botox were included to illustrate the rate at which song structure could change over $3 \mathrm{~d}$ (e.g., A, B). d27 AS, Accuracy score between songs at post-Botox day 27 and pre-Botox songs.

Song distortion compared with measures of new HVC neuron recruitment. To determine whether song distortion was correlated with new neuron recruitment and early survival, we compared the song accuracy scores at each sampling point (post-Botox days 7, 14, and 27) with our measures of new neurons in HVC: (1) the estimated total number of new neurons in HVC, (2) the percentage of new neurons of the total number of neurons in $\mathrm{HVC}$, and
(3) new neuron density per area sampled. New neuron recruitment and survival may be differentially influenced by experience depending on neuron age (Alvarez-Borda et al., 2004). Therefore, we were interested in determining whether correlations between the degree of song distortion and new neuron number were specific to particular times after Botox treatment, corresponding to different ages of our labeled neuron cohort. 
Rate of song recovery. For each bird, the rate at which the song recovered after Botox was determined by taking the difference between the pre-post accuracy scores at days 14 and 27 . These recovery scores were positive when post-Botox song became more similar to pre-Botox song over this interval and were negative when songs were less like pre-Botox songs at day 27 than at day 14 . We then compared the song recovery scores with our three measures of new neurons (listed above).

Rate of song recovery independent of the magnitude of distortion. We found that the rate of song recovery was positively correlated with prepost accuracy scores at post-Botox days 7, 14, and 27. Songs with the least distortion after Botox injection recovered fastest. Because distortion and recovery were related, we also assessed the correlation between recovery and new neurons independent of the degree of song distortion to determine whether recovery per se was correlated with new neuron number. To do this, the amount of song recovery between days 14 and 27 was divided by the amount of song distortion at day 14 . This resulted in a measure of song change between days 14 and 27 that was independent of the magnitude of distortion at day 14 . We then compared these independent song recovery scores with our measures of new neurons.

\section{Quantification of singing rate}

For each recording session, the number of motifs sung by each bird during the 13 daylight hours was counted, and a mean hourly rate was generated. To count motifs, we used the "Scan for Template Spectrogram Patterns" batch processing function in Avisoft SASLab Pro, which identifies and tallies occurrences of user-defined acoustic elements in a large database of recordings. In our case, we generated templates using syllable sequences unique to each bird's motif such that the sequences only occurred once in a motif and were not produced in the absence of a motif. The templates were between $\sim 0.15$ and $\sim 0.30 \mathrm{~s}$ long. Thus, the total number of sounds matching the template represented the number of motifs produced in a given database.

For control birds and experimental birds before Botox treatment, we also hand counted the number of motifs produced in a $2 \mathrm{~h}$ period and compared these motif counts with those generated by Avisoft during the same time period. The motif rates generated by the two methods were within \pm 5 motifs per hour ( 131 mean motifs per hour, maximum of $3.8 \%$ difference in counts between methods), and comparisons of singing rates and measures of new neurons based on hand-counted rates and computer-counted rates produced the same results. We report only the full-day computer-counted data in Results.

For some Botox-treated birds at day 7 after Botox injections, the high degree of song variability and noisy song structure restricted our ability to develop individual templates that would accurately identify motifs. Therefore, in these birds, post-Botox songs at day 7 were counted manually throughout the daylight hours. By days 14 and 27 after Botox injections, songs were stereotyped enough to count using Avisoft templates, and singing rates were confirmed manually for $2 \mathrm{~h}$ sample periods as described above.

Once individual singing rates were determined for particular days, we assessed reliability in singing rates across 4 days using a correlation matrix for four repeated measures. Then we compared singing rates between control and Botox birds at each of the four time periods (before Botox, post-Botox day 7 , post-Botox day 14 , and post-Botox day 27) to determine whether the Botox injections affected singing rate, and if so, whether particular time points after Botox reflected a greater effect of the treatment.

Next, we determined whether within the control group there was a correlation between singing rate and new neurons in HVC. For each control bird, we determined the average singing rate across the four sampling times. We then compared individual mean singing rates with the total number of new neurons in HVC, the percentage of new neurons in HVC, and new neuron density per square millimeter. This was done to identify whether there was a relation between singing and these metrics of new neuron recruitment in untreated control birds. We performed the same analyses within the Botox-treated group as with the controls: a comparison between new neurons and mean singing rate generated over all four recording days, and also independent comparisons between new neurons and singing rates at each of the four time periods. Finally, to complete the assessment of the potential association between singing rate and new neurons, we determined whether there were correlations between the change in individual singing rates between pre-Botox recordings and post-Botox days 7, 14, and 27 and our measures of new neurons in HVC.

\section{Selection of sampling times}

The recording days $(7,14$, and $27 \mathrm{~d}$ after cell birth dating) were selected to include time points in the lifespan of young neurons that encompassed different early life stages. By day 7, some new neurons are just arriving into HVC, but most of the labeled cohort has not yet arrived until day 14 (J.K., unpublished observations). At day 14, but not day 27, HVC neuron survival is sensitive to the growth factor BDNF (Alvarez-Borda et al., 2004), which is upregulated during singing (Li et al., 2000). HVC neuron recruitment/survival in canaries is sensitive to amount of singing between days 25 and 33 after cell birth dating (Li et al., 2000). To our knowledge, no other specific neuron ages have been shown to be sensitive to environmental or behavioral factors in songbirds, and therefore these time points represent our best guess as to when song structure or singing rate may influence HVC neuron survival or when new neurons may contribute to song recovery.

\section{Statistical analysis}

Data are presented as means \pm SEM unless noted otherwise. Analyses were performed using one-way ANOVAs and two-tailed $t$ tests for independent samples unless specified otherwise. Repeated-measures ANOVA was used to compare singing rates within individuals across recording days. Linear regression was used to assess relationships between behavioral and neural attributes. Kendall's tau rank correlation was used to assess inter-observer reliability in qualitative ranking of song spectrographs, and Spearman's rank correlation was used to evaluate correlations in rank orders of singing rates across days. All $p$ values are based on two-tailed tests.

\section{Results}

\section{Effects of Botox on song structure}

Injections of Botox into the syringeal muscles resulted in altered song acoustic structure that generally completely recovered by $\sim 4$ weeks (Fig. 2D). The most apparent effect of Botox was a noisy, fricative quality in syllable morphology and a change from frequency-modulated syllables to harmonic stacks (Fig. 2A-D). Frequency modulation is predominantly controlled by the paired muscles syringealis ventralis (Goller and Suthers, 1996), which received the bulk of the Botox injections; thus, loss of frequency control was predicted. There was individual variability in the effect of Botox on temporal measures (syllable duration and intersyllable intervals, as described by Pytte and Suthers, 2000).

Although care was taken to inject birds with identical dosages and in similar muscle locations, there was also variability in the severity of the effect of injections on song acoustic structure apparent 1 week after surgery as well as variability in the extent and rate of song recovery. The large range in individual responses to Botox and the individual variation in new HVC neuron numbers allowed for comparisons between these variables.

\section{Song stereotypy}

Although adult zebra finch song is generally stereotyped, there are individual differences in the stereotypy of fine structure (Pytte et al., 2007). Natural levels of song stereotypy were measured within individuals by comparing 30 pre-Botox motifs taken from $1 \mathrm{~d}$ with 30 motifs taken from a second pre-Botox day that was separated from the first by $3-5 \mathrm{~d}$. Songs were compared in a symmetrical matrix using Sound Analysis Pro, resulting in preto-pre Botox song accuracy scores, as described previously. The mean within-individual accuracy scores across the two pre-Botox days was 87.3 (range of 83-97), and this measure of individual 
A

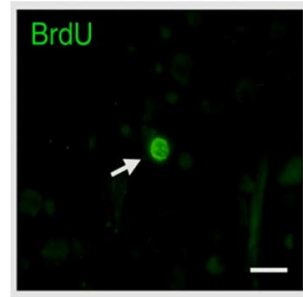

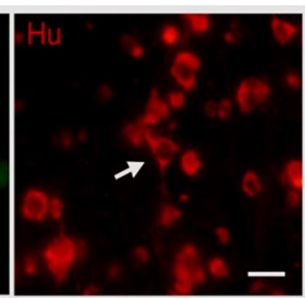

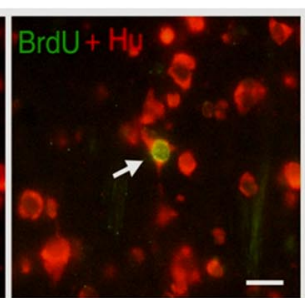

B

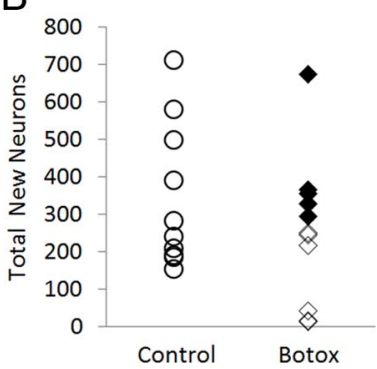

Figure 3. A, Photographs of the same field of view in HVC showing the fluorescent markers used to identify newly formed neurons. Arrow shows a BrdU-labeled nucleus (FITC filter), Hu-labeled cytoplasm (rhodamine filter), and colocalization of the two markers (dual filter). Scale bars, $10 \mu \mathrm{m}$. B , There was no difference in total new HVC neuron number sampled 1 month after cell birth dating between experimental and control groups $(p>0.05)$. Circles, Controls; filled diamonds, Botox-treated birds with high accuracy scores; open diamonds, Botox-treated birds with low accuracy scores.

song stereotypy did not predict the magnitude of distortion after Botox injections or the rate of song recovery $(p>0.05)$. PreBotox song stereotypy also was not correlated with any measures of new neurons or HVC volume ( $p>0.05$ for all comparisons). In all comparisons described below, we found the same significant correlations whether we used data that were normalized to pre-Botox song variability or not normalized. We show only normalized data.

Within control birds, there was no change in song stereotypy over the duration of the experiment. Within controls, the mean pre-to-pre song accuracy score was 83.4 (range of 79-87), and the mean pre-post song accuracy score was 83.1 (range of $80-86$ ) (paired $t$ test, $p>0.05$ ).

\section{Effects of Botox on new neurons}

There was no overall group difference in new neuron recruitment between Botox-injected and control birds measured as total new neurons in HVC, percentage new neurons in HVC, or new neurons per square millimeter (Fig. $3 B, p>0.05$ ). For all figures, we show only total new neurons in HVC. Although there was no overall treatment effect when comparing new neuron numbers for Botox and control groups, visual inspection of graphs plotting new neuron measures against song accuracy at $27 \mathrm{~d}$ after Botox (the day closest to the time the animals were killed for brain analyses) suggested that the Botox group was not homogeneous. Data points tended to cluster into two subgroupings by day 27 based on song accuracy (Fig. $4 A$, bottom). The data for Botox animals were therefore reanalyzed using hierarchical clustering (JMP, version 9.0; SAS). This procedure groups data points into clusters in a manner that minimizes the SD among points within a cluster (Ward method). Clusters of all possible sizes were then computed (from one cluster containing all data points to 11 clusters, with each cluster representing a single animal), yielding a dendrogram. The cluster analysis separated the Botox-treated animals into two subgroups based on song accuracy scores, and all measures of new neurons were compared between these subgroups. An overall ANOVA comparing the percentage of new neurons among controls and the two subgroups of Botox-treated birds yielded a significant main effect for group $\left(F_{(2,21)}=5.52\right.$; $p<0.015)$. Other measures of new neurons were nearly significant ( $p<0.08$ for all comparisons). For all measures of new neurons, an ANOVA between the two subgroups of Botox birds ( $n=5$ vs $n=6$ ) was significant (total new neurons, $F_{(1,9)}=6.20$; percentage new neurons, $F_{(1,9)}=7.62$; density new neurons, $F_{(1,9)}=$ $7.06 ; p<0.05$ for all). We next compared percentage new neuron number between the cluster of animals with the lowest accuracy scores and control animals and found that this subgroup of Botox animals had significantly lower new neuron numbers compared with controls $\left(F_{(1,17)}=9.52 ; p<0.007\right)$. Other measures of new neurons yielded $p$ values $<0.07$.

When we then used dendrogram split points that defined four clusters instead of two, the four subgroups were not all statistically unique. This analysis supports the notion that the Botox animals were not a homogeneous group and that a grouping procedure that minimizes subgroup variance yields two unique clusters of animals. To further explore the relationship between individual differences in Botox effects and variation in neuron addition, regression analyses were performed, comparing song accuracy scores at multiple time points after Botox to measures of neuron addition.

\section{Song distortion was inversely correlated with new neurons}

Although neuron recruitment was assessed at post-Botox day 27, we sought to identify whether distortion at different time points was correlated with new neurons. Song accuracy (the inverse of song distortion) between pre-Botox songs and post-Botox songs at days 7, 14, and 27 after injections was positively correlated with each of our measures of new neurons: (1) the estimated total number of new neurons in HVC (Fig. $4 A$, day $7, R^{2}=0.588, p<$ 0.01 ; day $14, R^{2}=0.436, p<0.05$; day $\left.27, R^{2}=0.567, p<0.01\right)$; (2) the percentage of new neurons of the total number of neurons in $\operatorname{HVC}$ (day 7, $R^{2}=0.537, p<0.05$; day $14, R^{2}=0.375, p<$ 0.05 ; day $27, R^{2}=0.591, p<0.01$ ); and (3) new neuron density per area sampled (day $7, R^{2}=0.502, p<0.05$; day $14, R^{2}=0.421$, $p<0.05$; day $\left.27 R^{2}=0.615, p<0.005\right)$. There were no significant correlations between accuracy scores of pre-to-post Botox song at days 7,14 , and 27 and HVC volume ( $p>0.05$ for all).

\section{Song recovery was positively correlated with new neuron recruitment}

Syringeal muscle recovery is apparent in spectrographs when the distinguishing noisy quality of song has disappeared and note acoustic structure is distinct and stereotyped (Fig. 2). By $27 \mathrm{~d}$ after Botox, song was either stabilized (Fig. $2 B, D$ ) or not yet stabilized (Fig. 2A,C), song had recovered (Fig. $2 D$ ), was reemerging but not completely recovered (Fig. $2 C$ ), or, in a few birds, the song that emerged during muscle recovery appeared to be different enough from the pre-Botox song to suggest a change in the underlying song motor program $(n=3$, two examples are shown in Fig. $2 A, B)$. Song stability is a qualitative description of the consistency in acoustic structure across motifs, which is apparent over bouts, hours, and days based on visual inspection of spectrographs. Muscle damage might result in poor-quality repro- 
A

Post-Botox Day 7

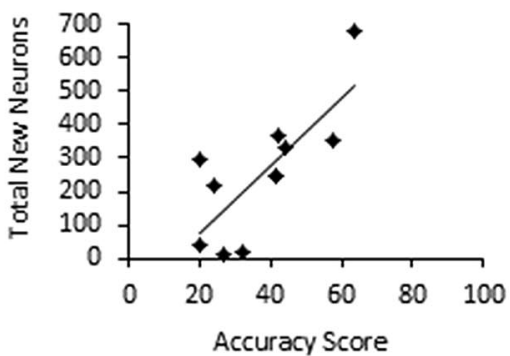

Post-Botox Day 14

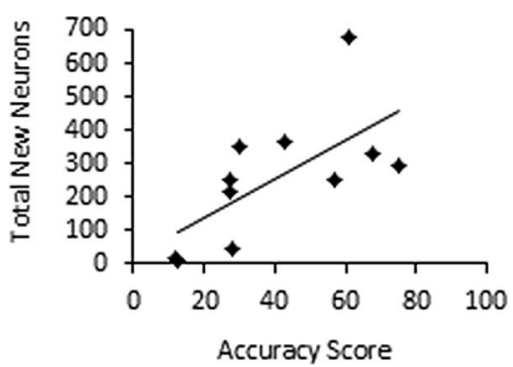

Post-Botox Day 27

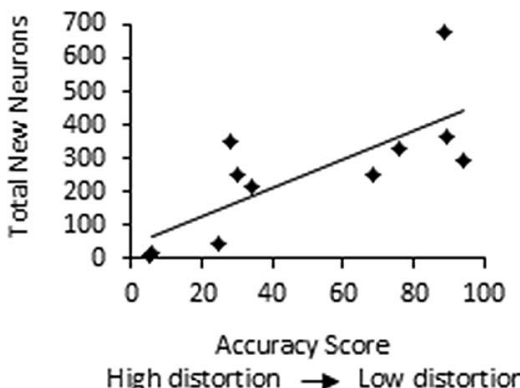

B

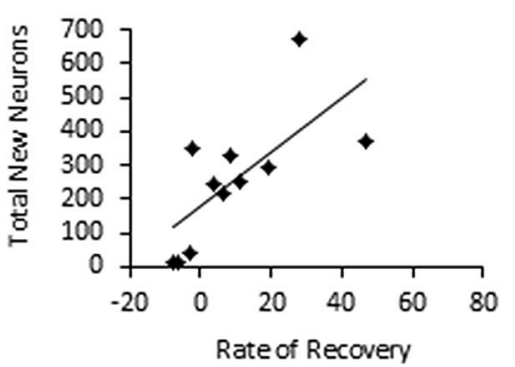

Figure 4. A, Accuracy scores between post-Botox songs and pre-Botox songs were positively correlated with total new neurons in $\operatorname{HVC}(p<0.05)$. Song accuracy was calculated as the mean accuracy index between 30 songs before Botox and 30 songs after Botox, normalized for pre-Botox variability using Sound Analysis Pro (Tchernichovski et al., 2000). Graphs depict the numbers of new neurons at the time the birds were killed, plotted against accuracy scores at post-Botox days 7, 14, and 27. B, The rate of song recovery between days 14 and 27 after Botox injection was positively correlated with the total estimate of 1 -month-old neurons in $\mathrm{HVC}(p<$ 0.05 ). Song recovery was measured as the difference in accuracy scores between pre-Botox songs and distorted songs at days 14 and 27 after Botox injections. More distorted songs tended to recover least over the period of study. Songs of four birds with the most song distortion at day 14 after Botox injections became increasingly less similar to their pre-Botox songs, seen here as negative values for song recovery.

ductions of the pre-Botox song but would likely not explain the selective deletion or addition of song elements apparent in changed temporal patterns. However, we cannot completely rule out a contribution of incomplete muscle recovery.
We first visually assessed song recovery by comparing spectrographs of pre-Botox songs and day 27 post-Botox songs. Spectrograms were visually scored by two sets of observers and ranked in ascending order of degree of distortion. Inter-observer reliability scores for the two ranks were significantly correlated (Kendall's tau $=8.182, p<0.001)$. Ranks were then averaged between the two sets of scores, and the average rank order was compared with rank orders for the measures of new neurons in HVC. We found a significant correlation between rank order of song recovery and rank order of total new neurons in HVC (Kendall's tau $=0.564, p<0.05)$ and rank order of new neurons per square millimeter (Kendall's tau $=0.600, p<0.05$ ) and a trend between rank order of song recovery and percentage new neurons in HVC (Kendall's tau $=0.418, p=0.087$ ). Rank order of song recovery was not significantly correlated with rank order of HVC volume ( $p>0.05)$.

For a more quantitative and sensitive analysis, the rate of song recovery was then measured using Sound Analysis software and calculated as the change in pre-to-post song accuracy scores between days 14 and 27 after Botox injections. We found positive correlations between the rate of song recovery during this period and the number of new neurons in HVC measured as the total number of new neurons in HVC (Fig. $4 B, R^{2}=0.474, p<0.05$ ), the percentage of new neurons in $\mathrm{HVC}\left(R^{2}=0.616, p<0.005\right)$, and new neurons per square millimeter $\left(R^{2}=0.592, p<0.01\right)$. There was no correlation between song recovery and HVC volume $\left(R^{2}=0.164, p>0.05\right)$.

\section{Song recovery as a function of the magnitude of distortion}

The rate of song recovery and magnitude of song distortion can be measured separately, and we found that they were not independent variables. The rate of song recovery between postoperative days 14 and 27 was positively correlated with the accuracy scores between pre- and post-Botox songs at postoperative days 7 $\left(R^{2}=0.402\right), 14\left(R^{2}=0.482\right)$, and $27\left(R^{2}=0.703\right)(p<0.05$ for all). Songs that were least distorted after Botox injections showed the fastest rate of song recovery and the highest magnitude of recovery. Post-Botox songs that were severely distorted showed the slowest rate of recovery and the lowest magnitude of recovery. These relationships are consistent with findings of dosedependent effects of Botox on motor behavior and recovery rates in other motor systems (Landers et al., 2002).

Because recovery and magnitude of distortion were correlated, we could not attribute our correlations between song recovery and measures of new neurons to song recovery per se. Therefore, we calculated the rate of song recovery independent of the magnitude of distortion (independent recovery rate) by normalizing the rate of recovery between 14 and $27 \mathrm{~d}$ using the value of song distortion at day 14 (see Materials and Methods).

The rate of recovery independent of the initial degree of song distortion was positively correlated with total new HVC neuron number $\left(R^{2}=0.474, p<0.05\right)$, the percentage of new HVC neurons $\left(R^{2}=0.625, p<0.005\right)$, and new neuron density per area of HVC sampled $\left(R^{2}=0.595, p<0.01\right)$. Thus, new neuron number was correlated with both the severity of song distortion and the rate of recovery. There was no correlation between independent recovery rate and HVC volume ( $p>0.05)$.

\section{Singing rate}

Because singing rate has been shown to be positively correlated with the number of 1-month-old neurons in the HVC of canaries (Li et al., 2000; Alvarez-Borda and Nottebohm, 2002), we assessed whether our correlations between song structure and new 


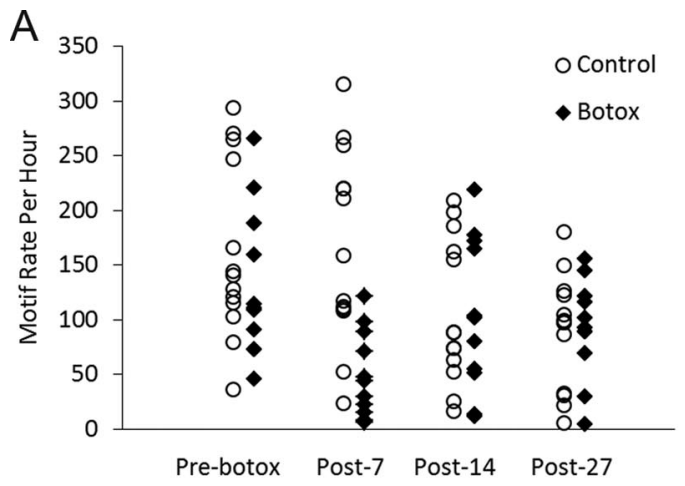

Figure 5. $A$, Control birds had a higher singing rate than Botox birds at day 7 after Botox injections $(p<0.01)$ and no group differences in singing rate before Botox or before sham or at posttreatment days 14 or $28(p>0.05)$. Singing rate was calculated as mean motifs per hour counted over a full daylight period ( $13 \mathrm{~h}$ ). $\boldsymbol{B}$, Within the control birds, there were no correlations between mean singing rate calculated over the four sample periods shown in $A$ and new neurons per HVC. Error bars show SEM for each bird.

neurons were in fact attributable to an effect of Botox on singing quantity rather than song quality or recovery.

We first determined whether relative differences in singing rates were conserved across the sampling periods before Botox or sham, post-Botox/sham day 7, post-Botox/sham day 14, and post-Botox/sham day 27. We found general consistency among controls and less so among Botox-treated birds. In controls, we found significant rank-order correlations among two pre-sham days $\left(r_{\mathrm{s}}=0.7697, p<0.005\right)$ and between pre-sham and post-sham day 7

$\left(r_{\mathrm{s}}=0.687, p<0.005\right)$, pre-sham and post-sham day $14\left(r_{\mathrm{s}}=\right.$ $0.614, p<0.05)$, and between post-sham days 7 and 14 $\left(r_{\mathrm{s}}=0.644, p<0.05\right)$. Post-day 27 singing rates were not correlated with any other days $(p>0.05)$.

Among Botox-treated birds, singing rank orders were correlated across two pre-Botox days $\left(r_{\mathrm{s}}=0.9451, p<0.005\right)$, postBotox days 14 and $27\left(r_{\mathrm{s}}=0.718, p<0.05\right)$, and between pre-Botox and post-Botox day $14\left(r_{\mathrm{s}}=0.591, p=0.05\right)$. Rank orders of singing rates across other days were not significantly correlated.

We then examined whether there were group differences in singing rates by comparing control and Botox birds at each of the four time periods: pre-Botox/sham, post-Botox day 7, postBotox day 14, and post-Botox $27 \mathrm{~d}$ after Botox/sham (Fig. 5A). We found a higher singing rate in control birds than Botox birds at day 7 after Botox injections (two-tailed $t$ test, $t=3.83$, $p<$ 0.01 ) and no difference in singing rates pre-Botox/sham or at days 14 or 27 (two-tailed $t$ test, $p>0.05$ ).

\section{Controls}

We next assessed whether there was a relation between singing rates and numbers of new neurons in HVC within the control group. There were no correlations between mean singing rates, calculated over the four sample periods shown in Figure $5 A$, and any of our measures of new neurons: total new neurons per HVC (Fig. $5 B$ ), percentage of new neurons of total neurons in HVC, or new neurons per square millimeter $(p>0.05$ for all). There was also no correlation between mean singing rate and HVC volume $(p>0.05)$. In case the mean singing rate obscured correlations between new neurons and singing only at specific time points, we also looked for a relation between new neurons and singing rates at days 7, 14, and 27 separately. We found no correlations between singing rates at any individual sampling time and any measures of new neurons or HVC volume $(p>0.05)$.
Botox-injected group

Within the Botox-treated group, we performed the same analyses as with the controls. Neither the overall mean singing rate for each bird (data not shown) nor the singing rates at individual sampling times were correlated with any measure of neuron addition ( $p>0.05$; Fig. 6). There was no correlation between singing rate at any time point and HVC volume $(p>$ 0.05). Finally, we determined that, within the Botox-treated group, there was no correlation between the change in singing rate between pre-Botox and post-Botox days 7, 14, and 27 and any measure of new neurons in HVC (nine comparisons, $p>$ 0.05 for all). It is possible that our sampling procedure was not sensitive enough to capture individual differences in the total amount of singing between the time of cell labeling and perfusion or at a particular time point that may have been critical to new neuron survival. However, correlations of singing rates across days suggest that our sampling procedures yielded singing rate estimates that were accurate for at least 2 week intervals.

\section{Discussion}

Here we demonstrate that the amount of song recovery after reversible syringeal paralysis was positively correlated with survival of new HVC neurons. Botox injections in the syrinx muscles resulted in individual variability in song distortion and rate and extent of recovery of pre-operative song structure. We found significant correlations between each of these variables and new neurons in HVC 1 month after cell birth dating. Birds with the greatest song integrity after Botox had the highest rate and magnitude of song recovery and the highest number of BrdU-labeled neurons. Singing amount did not predict new neuron survival. These results suggest that neuron addition in HVC is associated with the quality of song structure and motor progression toward a target song. There was no group difference in new neurons between control and Botox-treated birds. However, we found a bimodal difference in degree of song recovery by Botox-treated birds such that birds that showed the least recovery had significantly fewer new neurons than both controls and the subset of Botox-treated birds that recovered their song.

\section{Mechanisms of Botox effects}

Botulinum toxin type A (i.e., Botox) induces muscle paralysis by preventing acetylcholine release at the neuromuscular junction (Lebeda et al., 2008). Muscle recovery begins 10-28 d after Botox injection by sprouting accessory terminals (Wright et al., 2007), followed by recovery of the original inactivated synapse (de Paiva et al., 1999). Botox can be transported from muscle into the motor neuron and also to afferent neurons innervating the muscle spindle (Wiegand et al., 1976). Although syringeal muscle lacks muscle spindles (Wild, 2004, 2008), syringeal afference may be carried by an ascending component of the tracheosyringeal nerve to nucleus basalis (Bottjer and Arnold, 1982). Thus, it is possible that Botox was taken up by this population and also by syringeal motor neurons.

Transynaptic effects of Botox have been observed after injection directly into the brain (Antonucci et al., 2008). However, there is no evidence that Botox injected intramuscularly is trans- 
ported transynaptically to premotor or preafferent neurons, despite extensive research directed toward finding such effects (Currà et al., 2004; Antonucci et al., 2008). Central plasticity in motor patterns induced by intramuscular Botox in other systems has been consistently attributed to altered sensory feedback to the CNS rather than retrograde effects of injected Botox (Currà et al., 2004). Thus, altered sensory feedback after Botox may have influenced new neuron survival in the present work. However, our data do not identify the potential directionality of causation between new neuron numbers and song recovery, and we discuss two models below.

\section{Model A: neuron addition affects song structure}

In this model, neuron survival is not influenced by Botox or song structure. Instead, birds with high rates of neuron addition to HVC may be better able to compensate for muscle constraints resulting from Botox. Botox was injected in the ventral syringeal muscles, leaving dorsal, lateral, and perhaps some ventral muscle fibers responsive to motor commands. Neurons added to HVC in adulthood project to RA (Kirn et al., 1991; Scotto-Lomassese et al., 2007), and HVC-RA neurons appear to be responsible for the timing of distinct, 6-10 ms premotor commands (Hahnloser et al., 2002). Perhaps newly incorporated HVC-RA neurons provided increased degrees of freedom for motor commands to adjust to the partial paralysis. New neurons may be engaged in exploratory activity in an attempt to regain the original song pattern, and thus song recovery may reflect this plasticity in addition to the reversal of paralysis. If so, HVC compensation for syringeal paralysis is evident 1 week after Botox when we see a correlation between song structure and new neuron number. At this stage, HVC plasticity would be using neurons present before the arrival of our labeled cohort.

Scharff et al. (2000) determined that selective ablation of HVC-RA neurons resulted in song deterioration in four of nine experimental birds. Although they found no difference in neuron recruitment in HVC between birds with song deterioration and those without, they reported a trend toward greater neuronal incorporation associated with greater improvement in syllable sequence (syllable morphology was not analyzed quantitatively). Both these findings are consistent with our results. Perhaps naturally high numbers of new neurons facilitate recovery of the original song, and failure to recover pre-Botox song is the result of limited neural resources.

\section{Model B: song structure affects neuron addition}

Alternatively, recovery of the pre-Botox song might be solely the result of peripheral recovery from Botox. Variability in song distortion and recovery may be attributable to differences in extent of paralysis resulting from variation in Botox injections (Landers et al., 2002) and not preexisting differences in neuron recruitment. By altering song at the periphery, we may be experimentally provoking the system to respond as if the song motor
Pre-Botox

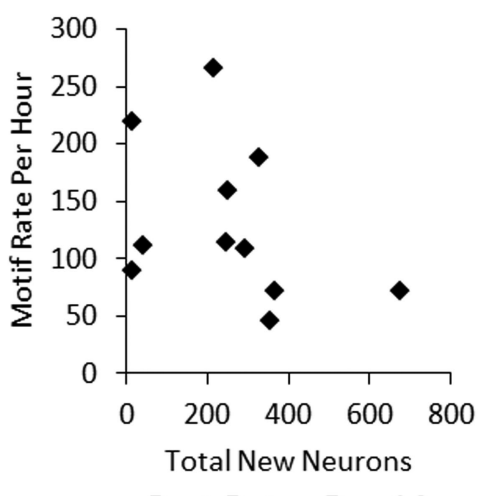

Post-Botox Day 14

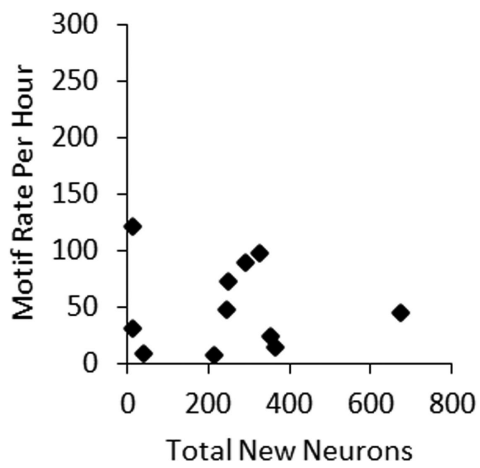

Total New Neurons
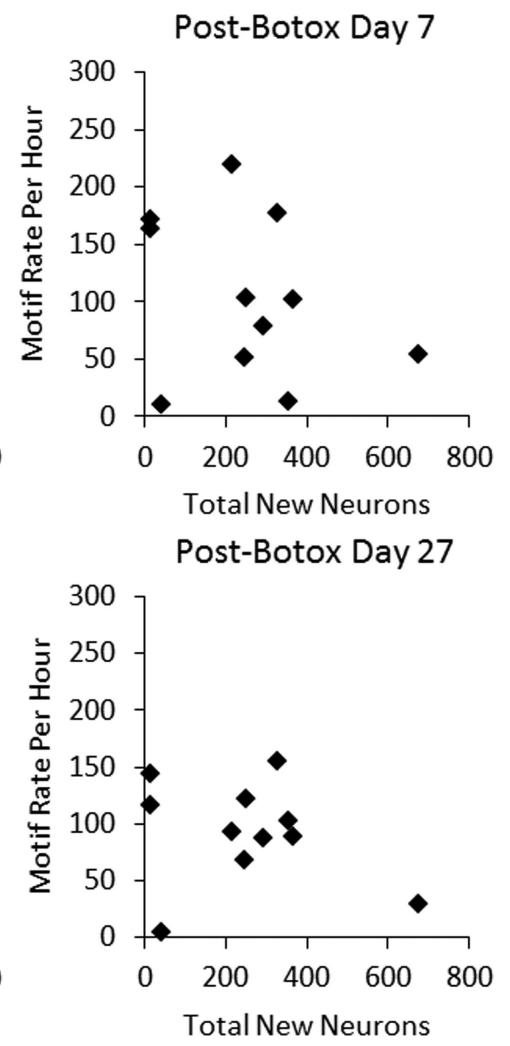

Figure 6. Within the Botox-treated group, there were no correlations between singing rate at any of the four sampling times (pre-Botox, post-Botox days 7, 14, and 27) and any of our measurements of new neurons (total new neurons per HVC, percentage of new neurons, density of new neurons, $p>0.05$ for all).

program is flawed, resulting in feedback-based culling of the labeled population of incoming HVC neurons in proportion to the degree of song distortion. Moreover, selective retention of incoming neurons may also be based on the contribution they make to song improvement per se rather than song quality at a given time. This process would allow best-fit neurons to be maintained and accumulate in HVC as long as song feedback indicated increasingly accurate song.

Feedback-related neuron culling could be based on an active error signal (Brainard and Doupe, 2001) or a lack of reinforcement or reward signal (Doupe et al., 2005). One trophic molecule that could be associated with error/reward signals is BDNF (Horger et al., 1999; Graham et al., 2007; Beste et al., 2010; McHughen et al., 2010). There is a natural culling of new HVC neurons between 15 and $22 \mathrm{~d}$ after cell birth dating, during the time many of these cells are first establishing connections in RA (Kirn et al., 1999). At this time, there is also a critical period during which BDNF exposure enhances new HVC neuron survival (Alvarez-Borda et al., 2004). In the mammalian striatum, reward increases BDNF expression (Zhang et al., 2002). Perhaps distorted feedback, or error signals, diminish BDNF expression and, in turn, the survivorship of new neurons. Likewise, reward attributable to increasingly accurate singing may increase BDNF and neuron survival.

\section{Singing and new neurons}

Suppression of singing in adult canaries for $8 \mathrm{~d}$ before the birds were killed resulted in decreased new neuron survival in HVC compared with freely singing birds ( $\mathrm{Li}$ et al., 2000). However, a comparison between individual singing rates and new neurons, a measure directly comparable with our study, has not been re- 
ported. Alvarez-Borda and Nottebohm (2002) showed a correlation between singing amount and new neurons in castrated male canaries but not in intact males. Over an annual time span in canaries, seasonal variation in singing and new neuron addition to HVC show an inverse relationship. HVC neuron addition is highest in the fall when singing rate is low, before the establishment of stereotyped song (Kirn et al., 1994). This suggests that factors other than amount of song, perhaps including song quality, are important (Boseret et al., 2006). It would be interesting to see whether song quality influences BDNF expression in zebra finches and also in seasonal singers. We do not know whether song quality influences testosterone or its metabolites, but these trophic factors have also been shown to modulate HVC neuron survival (Rasika et al., 1994; Alvarez-Borda and Nottebohm, 2004; Ball et al., 2004; Balthazart et al., 2010; Thompson and Brenowitz, 2010) and to respond to behavioral feedback (Sartor et al., 2005).

\section{Conclusions}

Early developmental or genetically determined rates of new neuron addition to HVC persist into adulthood, even after deafening, arguing for strong experience-independent factors governing neuron incorporation (Hurley et al., 2008). This, plus our finding of no group differences in neuron addition between Botox and control groups is consistent with model A. We also found in post hoc analyses that the Botox-treated group could be subdivided based on severity of song distortion, and the subgroup with greater song distortion had significantly fewer new neurons than remaining Botox-treated birds or controls. This is consistent with either model but argues that feedback must be severely aberrant if it is to have an effect on neuron survival. Thus, collectively, our results favor model $\mathrm{A}$. Of course, the two models need not be mutually exclusive and may comprise a feedback loop. Birds with naturally high rates of neuron addition may be at an advantage regarding vocal exploratory behavior necessary for song recovery, and behavioral recovery may encourage neuron survival. The possibility that naturally occurring variation in new neuron number affects song recovery could perhaps be directly tested by suppressing neurogenesis with an antimitotic agent, radiation, or other means and determining whether this decreases rate of song recovery after Botox. Future work should aim to address the functional directionality of this association.

\section{References}

Alvarez-Borda B, Nottebohm F (2002) Gonads and singing play separate, additive roles in new neuron recruitment in adult canary brain. J Neurosci 22:8684-8690.

Alvarez-Borda B, Haripal B, Nottebohm F (2004) Timing of brain-derived neurotrophic factor exposure affects life expectancy of new neurons. Proc Natl Acad Sci U S A 101:3957-3961.

Alvarez-Buylla A, Kirn JR, Nottebohm F (1990) Birth of projection neurons in adult avian brain may be related to perceptual or motor learning. Science 249:1444-1446.

Antonucci F, Rossi C, Gianfranceschi L, Rossetto O, Caleo M (2008) Longdistance retrograde effects of botulinum neurotoxin A. J Neurosci 28:3689-3696.

Ball GF, Auger CJ, Bernard DJ, Charlier TD, Sartor JJ, Riters LV, Balthazart J (2004) Seasonal plasticity in the song control system: multiple brain sites of steroid hormone action and the importance of variation in song behavior. Ann N Y Acad Sci 1016:586-610.

Balthazart J, Charlier TD, Barker JM, Yamamura T, Ball GF (2010) Sex steroid-induced neuroplasticity and behavioral activation in birds. Eur J Neurosci 32:2116-2132.

Beste C, Kolev V, Yordanova J, Domschke K, Falkenstein M, Baune BT, Konrad C (2010) The role of the BDNF Val66Met polymorphism for the synchronization of error-specific neural networks. J Neurosci 30:10727-10733.

Boseret G, Carere C, Ball GF, Balthazart J (2006) Social context affects testosterone-induced singing and the volume of song control nuclei in male canaries (Serinus canaria). J Neurobiol 66:1044-1060.

Bottjer SW, Arnold AP (1982) Afferent neurons in the hypoglossal nerve of the zebra finch (Poephila guttata): localization with horseradish peroxidase. J Comp Neurol 210:190-197.

Brainard MS, Doupe AJ (2001) Postlearning consolidation of birdsong: stabilizing effects of age and anterior forebrain lesions. J Neurosci 21:2501-2517.

Clark SJ, Cynx J, Alvarez-Buylla A, O’Loughlin B, Nottebohm F (1990) On variables that affect estimates of the true sizes and densities of radioactively labeled cell nuclei. J Comp Neurol 301:114-122.

Currà A, Trompetto C, Abbruzzese G, Berardelli A (2004) Central effects of botulinum toxin type A: evidence and supposition. Mov Disord 19 [Suppl 8]:S60-S64.

de Paiva A, Meunier FA, Molgó J, Aoki KR, Dolly JO (1999) Functional repair of motor endplates after botulinum neurotoxin type A poisoning: biphasic switch of synaptic activity between nerve sprouts and their parent terminals. Proc Natl Acad Sci U S A 96:3200-3205.

Derégnaucourt S, Mitra PP, Fehér O, Pytte C, Tchernichovski O (2005) How sleep affects the developmental learning of bird song. Nature 433:710-716.

Doupe AJ, Perkel DJ, Reiner A, Stern EA (2005) Birdbrains could teach basal ganglia research a new song. Trends Neurosci 28:353-363.

Goller F, Suthers RA (1996) Role of syringeal muscles in controlling the phonology of bird song. J Neurophysiol 76:287-300.

Graham DL, Edwards S, Bachtell RK, DiLeone RJ, Rios M, Self DW (2007) Dynamic BDNF activity in nucleus accumbens with cocaine use increases self administration and relapse. Nat Neurosci 10:1029-1037.

Guillery RW, Herrup K (1997) Quantification without pontification: choosing a method for counting objects in sectioned tissues. J Comp Neurol 386:2-7.

Hahnloser RH, Kozhevnikov AA, Fee MS (2002) An ultra-sparse code underlies the generation of neural sequences in a songbird. Nature 419:65-70.

Horger BA, Iyasere CA, Berhow MT, Messer CJ, Nestler EJ, Taylor JR (1999) Enhancement of locomotor activity and conditioned reward to cocaine by brain-derived neurotrophic factor. J Neurosci 19:4110-4122.

Hurley P, Pytte CL, Kim JR (2008) Nest of origin predicts adult neuron addition rates in the vocal control system of the zebra finch. Brain Behav Evol 71:263-270.

Kirn JR, DeVoogd TJ (1989) Genesis and death of vocal control neurons during sexual differentiation in the zebra finch brain. J Neurosci 9:3176-3187.

Kirn JR, Alvarez-Buylla A, Nottebohm F (1991) Production and survival of projection neurons in a forebrain vocal center of adult male canaries. J Neurosci 11:1756-1762.

Kirn JR, Fishman Y, Sasportas K, Alvarez-Buylla A, Nottebohm F (1999) Fate of new neurons in adult canary high vocal center during the first 30 days after their formation. J Comp Neurol 411:487-494.

Kirn J, O'Loughlin B, Kasparian S, Nottebohm F (1994) Cell death and neuronal recruitment in the high vocal center of adult male canaries are temporally related to changes in song. Proc Natl Acad Sci U S A 91:7844-7848.

Landers M, Pytte C, Zeigler HP (2002) Reversible blockade of rodent whisking: botulinum toxin as a tool for developmental studies. Somatosens Mot Res 19:358-363.

Lebeda FJ, Adler M, Erickson K, Chushak Y (2008) Onset dynamics of type A botulinum neurotoxin-induced paralysis. J Pharmacokinet Pharmacodyn 35:251-267.

Li XC, Jarvis ED, Alvarez-Borda B, Lim DA, Nottebohm F (2000) A relationship between behavior, neurotrophin expression, and new neuron survival. Proc Natl Acad Sci U S A 97:8584-8589.

McHughen SA, Rodriguez PF, Kleim JA, Kleim ED, Marchal Crespo L, Procaccio V, Cramer SC (2010) BDNF Val66Met polymorphism influences motor system function in the human brain. Cereb Cortex 20:1254-1262.

Nottebohm F, O’Loughlin B, Gould K, Yohay K, Alvarez-Buylla A (1994) The life span of new neurons in a song control nucleus of the adult canary brain depends on time of year when these cells are born. Proc Natl Acad Sci U S A 91:7849-7853. 
Pytte CL, Suthers RA (2000) Sensitive period for sensorimotor integration during vocal motor learning. J Neurobiol 42:172-189.

Pytte CL, Gerson M, Miller J, Kirn JR (2007) Increasing stereotypy in adult zebra finch song correlates with a declining rate of adult neurogenesis. Dev Neurobiol 67:1699-1720.

Rasika S, Nottebohm F, Alvarez-Buylla A (1994) Testosterone increases the recruitment and/or survival of new high vocal center neurons in adult female canaries. Proc Natl Acad Sci U S A 91:7854-7858.

Rasika S, Alvarez-Buylla A, Nottebohm F (1999) BDNF mediates the effects of testosterone on the survival of new neurons in an adult brain. Neuron 22:53-62.

Saper CB (1996) Any way you cut it: a new journal policy for the use of unbiased counting methods. J Comp Neurol 364:5.

Sartor JJ, Balthazart J, Ball GF (2005) Coordinated and dissociated effects of testosterone on singing behavior and song control nuclei in canaries ( $\mathrm{Se}$ rinus canaria). Horm Behav 47:467-476.

Scharff C, Kirn JR, Grossman M, Macklis JD, Nottebohm F (2000) Targeted neuronal death affects neuronal replacement and vocal behavior in adult songbirds. Neuron 25:481-492.

Scotto-Lomassese S, Rochefort C, Nshdejan A, Scharff C (2007) HVC interneurons are not renewed in adult male zebra finches. Eur J Neurosci 25:1663-1668.

Tchernichovski O, Nottebohm F, Ho CE, Pesaran B, Mitra PP (2000) A procedure for an automated measurement of song accuracy. Anim Behav 59:1167-1176.

Thompson CK, Brenowitz EA (2010) Neuroprotective effects of testoster- one in a naturally occurring model of neurodegeneration in the adult avian song control system. J Comp Neurol 518:4760-4770.

Tramontin AD, Brenowitz EA (1999) A field study of seasonal neuronal incorporation into the song control system of a songbird that lacks adult song learning. J Neurobiol 40:316-326.

Wiegand H, Erdmann G, Wellhöner HH (1976) ${ }^{125}$ I-labelled botulinum A neurotoxin: pharmacokinetics in cats after intramuscular injection. Naunyn Schmiedebergs Arch Pharmacol 292:161-165.

Wilbrecht L, Kirn JR (2004) Neuron addition and loss in the song system: regulation and function. Ann N Y Acad Sci 1016:659-683.

Wilbrecht L, Crionas A, Nottebohm F (2002) Experience affects recruitment of new neurons but not adult neuron number. J Neurosci $22: 825-831$.

Wild JM (2004) Functional neuroanatomy of the sensorimotor control of singing. Ann N Y Acad Sci 1016:438-462.

Wild JM (2008) Birdsong: anatomical foundations and central mechanisms of sensorimotor integration. In: Neuroscience of birdsong (Zeigler HP, Marler P, eds), pp 136-152. New York: Cambridge UP.

Williams H, McKibben JR (1992) Changes in stereotyped central motor patterns controlling vocalization are induced by peripheral nerve injury. Behav Neural Biol 57:67-78.

Wright MC, Cho WJ, Son YJ (2007) Distinct patterns of motor nerve terminal sprouting induced by ciliary neurotrophic factor vs. botulinum toxin. J Comp Neurol 504:1-16.

Zhang D, Zhang L, Lou DW, Nakabeppu Y, Zhang J, Xu M (2002) The dopamine D1 receptor is a critical mediator for cocaine-induced gene expression. J Neurochem 82:1453-1464. 